MATHEMATICS OF COMPUTATION

Volume 68, Number 227, Pages 1311-1315

S 0025-5718(99)01065-0

Article electronically published on February 13, 1999

\title{
NEW MAXIMAL PRIME GAPS AND FIRST OCCURRENCES
}

\author{
THOMAS R. NICELY
}

\begin{abstract}
The search for first occurrences of prime gaps and maximal prime gaps is extended to $10^{15}$. New maximal prime gaps of 806 and 906 are found, and sixty-two previously unpublished first occurrences are found for gaps varying from 676 to 906 .
\end{abstract}

\section{INTRODUCTION}

The study of the distribution of the prime numbers among the positive integers occupies a central place in number theory. This distribution may be specified by the differences $G_{k}=p_{k+1}-p_{k}$ between successive primes; $G_{k}$ is also referred to as the (prime) gap following the $k$ th prime $p_{k}$, and the unmodified symbol $G$ will be used to refer to a specific gap as well as the set of all prime gaps of a specified magnitude. A gap $G$ contains $G-1$ consecutive composite integers. All gaps are even positive integers except for $G_{1}=1$; it is an open question whether or not gaps of magnitude $2 n$ exist corresponding to each and every positive integer $n$. The "first occurrence" of a gap $G$ is defined by the smallest prime $p_{k}$ preceding (immediately followed by) such a gap. A (first occurrence of a) gap $G$ is said to be "maximal" if all preceding gaps (between smaller consecutive primes) are strictly less than $G$. Thus the first occurrence of a gap of 10 follows the prime 139, but this is not a maximal gap, since an equal or larger gap (a maximal gap of 14, following 113) appears earlier in the sequence of positive integers. Note that some authors (e.g., Riesel $\left[14\right.$, p. 80]) specify first occurrences by the terminating prime $p_{k+1}$, while others specify a gap by the parameter $r=G / 2$ (e.g., Brent $[2,3]$ ).

No general method more sophisticated than an exhaustive search is known for the determination of first occurrences and maximal prime gaps. As in the present study, this is most efficiently done by sieving successive blocks of positive integers for primes, recording the successive differences, and thus determining directly the first occurrences and maximal gaps. This technique has been used by Shanks [15], Lander and Parkin [10], Brent [2,3], and Young and Potler [17] to extend the search through all primes $<7.263512 \times 10^{13}$. Thus all first occurrences of gaps through 674 , as well as scattered first occurrences for gaps through 778 , were tabulated, and all maximal prime gaps through 778 were located. See Young and Potler [17] for an exhaustive listing of these previous results. In addition, Young and Potler continued their calculations to an unpublished higher level; Ribenboim [13, p. 142]

Received by the editor June 16, 1997 and, in revised form, December 5, 1997.

1991 Mathematics Subject Classification. Primary 11A41; Secondary 11-04, 11Y11, 11Y99.

Key words and phrases. Prime numbers, prime gaps, first occurrences, maximal gaps, maximal prime gaps. 
credits them with the discovery of an additional maximal prime gap of 804 following the prime 90874329411493 , and this was confirmed by Young in [18].

Isolated occurrences of much larger prime gaps have been found. It is well known that arbitrarily large gaps exist, for the positive integer $(n !+1)$ must be followed by at least $(n-1)$ consecutive composite integers; but no instance of this formula beyond $n=5$ (first occurrence and maximal gap of 14 following 113) is known to yield a first occurrence. Weintraub [16] has discovered a gap of 864 following 6505941701960039, and Baugh and O'Hara [1] discovered a gap of 4248 following $10^{314}-1929$, but these are not known or believed to be maximal gaps or even first occurrences; the present work demonstrates conclusively that Weintraub's gap is not maximal. In conjunction with the search for seven consecutive primes in arithmetic progression [9], Dubner [7] has discovered a gap of 1092 following the prime 409534375009657239721; this is the first known occurrence of a gap of 1000 or greater, but again it is not known to be maximal or a first occurrence. Dubner in [8] also reports a gap of 12540 following the 385 -digit prime:

1028115851618596629291338345969573325611755920349536050557212232499 _

$6950065379512197585317961759000690328913319244717897688019822063737812_{-}$ $5686339726137874956095491930654497693978715833794999935477468391789508_{\text {_ }}$ 3444495414063479003554272907008549459458538251939796513140998638325548 _ 2457633841427250249367844894786016514356294279402896163593801089250404 _ 09462881632270278716570882306451587569 .

\section{Computational technique}

The search for prime gaps is being carried out as part of a larger program [11], which includes the enumeration of the primes, twin primes, prime triplets, and prime quadruplets for $0\left(10^{9}\right) 10^{14}$ and for $10^{14}\left(10^{10}\right) 10^{15}$ and beyond. Also computed are the floating point (64-bit mantissa) sums and ultraprecision (53 decimal places) sums of the reciprocals of the twins, triplets, and quadruplets, in order to extrapolate estimates for the Brun's constants (limits of the sums of the reciprocals) for each constellation. All computations are being executed during slack hours on personal computers belonging to the author or assigned to the Department of Mathematics at Lynchburg College. The number of systems (most of them Pentiums) in use has averaged about fifteen since the present program began in 1993. The source code is written in $\mathrm{C}$ and compiled using Borland $\mathrm{C}++4.52$; it is written to run under Borland's 32-bit DOS extender so that all available extended memory can be used for the integer arrays. Earlier versions ran successively under DOS, Windows 3.x, and Win 32s, but operation under 32-bit extended DOS proved most effective, eliminating much of the irrelevant overhead imposed by the Windows environment. Typical throughput is about $10^{11}$ integers per day on a $60 \mathrm{MHz}$ Pentium. The computations are distributed independently across the systems, currently in runs

of $2 \times 10^{12}$; each interval is run in duplicate on two systems to guard against machine errors. In the event that two runs of the same interval disagree, additional runs are carried out to resolve the discrepancy. Thus far, errors have been detected in nearly two dozen instances, including the Pentium FDIV flaw affair [12] in the fall of 1994; faults in memory chips appear to be the most frequent culprit, although it appears impossible to completely rule out errors in either system or application software. The most significant independent check available is the value of $\pi(x)$, the count 
of primes, which has been carried out by indirect means to $10^{20}$ by Deleglise and Rivat $[6,4]$; the direct counts obtained from the present calculations agree through $10^{15}$ with the values published by Riesel [14, p. 34 and pp. 380-383]. The first occurrences listed have also been checked directly by means of the Derive software program for DOS and Windows.

It is of interest to note that two of the Pentiums in service are P5-60 systems with (FDIV) flawed CPUs; the flawed floating point divisions and remainders are being detected and corrected in real time, using a combination of the $-\mathrm{fp}$ switch in Borland $\mathrm{C}++4.52$ and a custom procedure (C function) which traps suspect divisors in all fmod and fmodl remaindering calls. With these errors trapped and corrected, and their results checked against runs on CPUs free of the flaw, these two systems have remained error free for more than a year.

\section{Computational Results}

Table 1 lists the first occurrences of prime gaps found in the present study, now complete to $10^{15}$. The new maximal gaps are marked with an asterisk $\left(^{*}\right)$. As was pointed out above, the first occurrence and maximal gap of 804 following the prime 90874329411493 is actually due to Young and Potler [13, p. 142] and is confirmed by the present work. Presumably, the other first occurrences between $7.2 \times 10^{13}$ and $9.1 \times 10^{13}$ (for the gaps of $676,680,686$, and 718 ) were also known to Young and Potler, but were never published.

These results supplement those previously known and herein omitted for brevity; an exhaustive listing of previously known gaps was given by Young and Potler [17]. The smallest gap whose first occurrence is still unaccounted for is the gap of 796 . First occurrences of all gaps greater than 796, not listed in Table 1, also remain to be discovered. Discovery of the new maximal gap of 906 brings us closer to the goal alluded to by Weintraub [16], that of finding the first occurrence of a gap of 1000 or greater. Motivated by a result obtained by Cramér [5], Shanks [15] conjectured that a maximal gap of magnitude $M$ could be expected to appear at approximately $e^{\sqrt{M}}$; Riesel $[14$, p. 80] measures the success of this conjecture by the ratio $R=\ln \left(p_{k+1}\right) / \sqrt{M}$, with $R$ expected to approach 1 as $M$ and $p_{k+1}$ increase without bound. For the largest known maximal gaps, $R$ has remained near 1.13, although for the new maximal gap of 906 it attains a value of 1.0969 , its absolute minimum to date. Thus, assuming that the first gap of 1000 or greater will actually be about 1050, a reasonable estimate for the location of the first occurrence of a gap of 1000 or greater would be $e^{1.13 \sqrt{1050}} \approx 7.98 \times 10^{15}$. The present program would not attain that level for several more years. However, this is little more than an order of magnitude estimate, since an argument could also be made for a much smaller value of $e^{1.0969 \sqrt{1000}} \approx 1.16 \times 10^{15}$. The discovery of the first "kilogap" thus remains difficult to anticipate. 
TABLE 1. First occurrences of prime gaps in $7.2 \times 10^{13}<p<10^{15}$

\begin{tabular}{rrrr} 
Gap & \multicolumn{1}{c}{$\begin{array}{c}\text { Following } \\
\text { the prime }\end{array}$} & Gap & \multicolumn{1}{c}{$\begin{array}{c}\text { Following } \\
\text { the prime }\end{array}$} \\
& & & \\
676 & 78610833115261 & 782 & 726507223559111 \\
680 & 82385435331119 & 784 & 497687231721157 \\
686 & 74014757794301 & 786 & 554544106989673 \\
688 & 110526670235599 & 788 & 96949415903999 \\
704 & 97731545943599 & 790 & 678106044936511 \\
708 & 143679495784681 & 792 & 244668132223727 \\
710 & 138965383978937 & 794 & 673252372176533 \\
712 & 106749746034601 & 798 & 309715100117419 \\
718 & 82342388119111 & 800 & 486258341004083 \\
720 & 111113196467011 & 802 & 913982990753641 \\
722 & 218356872845927 & $804^{*}$ & 90874329411493 \\
726 & 156100489308167 & $806^{*}$ & 171231342420521 \\
732 & 140085225001801 & 808 & 546609721879171 \\
734 & 154312610974979 & 810 & 518557948410967 \\
736 & 161443383249583 & 814 & 827873854500949 \\
738 & 143282994823909 & 816 & 632213931500513 \\
742 & 189442329715069 & 818 & 860149012919321 \\
746 & 184219698008123 & 820 & 497067290087413 \\
748 & 172373989611793 & 822 & 799615339016671 \\
750 & 145508250945419 & 826 & 407835172832953 \\
752 & 255294593822687 & 828 & 807201813046091 \\
754 & 219831875554399 & 830 & 507747400047473 \\
760 & 98103148488133 & 832 & 243212983783999 \\
762 & 144895907074481 & 834 & 743844653663833 \\
764 & 323811481625339 & 836 & 880772773476623 \\
768 & 423683030575549 & 840 & 670250273356109 \\
770 & 214198375528463 & 844 & 782685877447783 \\
772 & 186129514280467 & 860 & 844893392671019 \\
774 & 469789142849483 & 862 & 425746080787897 \\
776 & 187865909338091 & 872 & 455780714877767 \\
780 & 471911699384963 & 880 & 277900416100927 \\
& & $906 *$ & 218209405436543
\end{tabular}

*Maximal gap.

\section{Acknowledgments}

The author wishes to express his appreciation to Lynchburg College for permitting the use of the departmental computers; to the Lynchburg College reference librarians for their untiring assistance; to Richard P. Brent and the late Daniel Shanks for their advice and encouragement; to Intel Corporation for the donation of computer systems and processors; to Intel's engineers, particularly Bob Davies and Dave Papworth, for their assistance in optimizing code for the Pentium Pro; to Arjen Lenstra, whose ultraprecision routines I modified for use in my code; to Jörg 
Richstein, Universität Giessen, Germany, for recommending and encouraging the addition of this line of investigation; and to the anonymous referee for his helpful suggestions.

\section{REFERENCES}

1. D. Baugh and F. O'Hara, Letters to the Editor, Large Prime Gaps and and More, J. Recreational Math 24:3 (1992) 186-187.

2. R. P. Brent, The first occurrence of large gaps between successive primes, Math. Comp. 27:124 (1973), 959-963. MR 48:8360

3. R. P. Brent, The first occurrence of certain large prime gaps, Math. Comp. 35:152 (1980), 1435-1436. MR 81g:10002

4. C. Caldwell, The Prime Page, at http://www.utm.edu/research/primes/.

5. H. Cramér, On the order of magnitude of the difference between consecutive prime numbers, Acta Arith. 2 (1937), 23-46.

6. M. Deleglise and J. Rivat, Computing $\pi(x)$ : The Meissel, Lehmer, Lagarias, Miller, Odlyzko Method, Math. Comp. 65 (1996), 235-245. MR 96d:11139

7. H. Dubner, private e-mail communication, 4 August 1996.

8. H. Dubner, private e-mail communication, 2 September 1996.

9. H. Dubner and H. Nelson, Seven consecutive primes in arithmetic progression, Math. Comp. 66 (1997), 1743-1749. MR 98a:11122

10. L. J. Lander and T. R. Parkin, On the first appearance of prime differences, Math. Comp. 21 (1967), 483-488. MR 37:6237

11. T. R. Nicely, Enumeration to $10^{14}$ of the twin primes and Brun's constant, Virginia Journal of Science 46:3 (1995), 195-204. MR 97e:11014

12. T. R. Nicely, unpublished document, available at http://www.lynchburg.edu/public/academic/math/nicely/pentbug/pentbug.txt.

13. P. Ribenboim, The little book of big primes, Springer-Verlag, New York, 1991. MR 92i:11008

14. H. Riesel, Prime numbers and computer methods for factorization, 2nd ed., Birkhäuser, Boston, 1994. MR 95h:11142

15. D. Shanks, On maximal gaps between successive primes, Math. Comp. 18 (1964), 646-651. MR 29:4745

16. S. Weintraub, A prime gap of 864, J. Recreational Math. 25:1 (1993), 42-43.

17. J. Young and A. Potler, First occurrence prime gaps, Math. Comp. 52:185 (1989), 221-224. MR 89f: 11019

18. J. Young, private e-mail communication, 6 June 1996.

Department of Mathematics, Lynchburg College, 1501 Lakeside Drive, Lynchburg, VIRGINIA 24501-3199

E-mail address: nicely@acavax.lynchburg.edu

URL: http://www.lynchburg.edu/public/academic/math/nicely/index.htm 